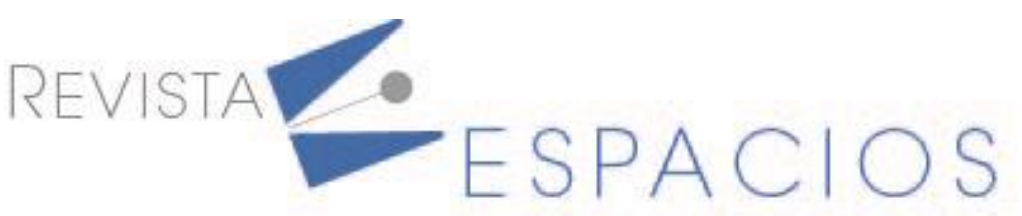

Vol. 41 (43) $2020 \cdot$ Art. 1

Recibido/Received: 28/02/2020 • Aprobado/Approved: 15/09/2020 • Publicado/Published: 12/11/2020

\title{
Valor compartido y planeación estratégica: factores de transformación empresarial en el sector minero
}

\section{Shared value and strategic planning: factors of business transformation in the mining sector}

\author{
BEDOYA JIMÉNEZ, Mauricio A. ${ }^{1}$ \\ MARTÍNEZ GÓMEZ, Jormaris. ${ }^{2}$ \\ LÓPEZ-MEDINA, Juan-D. ${ }^{3}$ \\ QUINTERO ARANGO, Luis F. ${ }^{4}$
}

\begin{abstract}
Resumen
La intención de este artículo fue identificar cómo la empresa minera DG1 vincula, desde la planeación estratégica y el valor compartido, su propuesta de negocio frente a la comunidad interesada. La metodología utilizada en la investigación fue cualitativa, descriptiva, no experimental. Los resultados encontrados establecieron que definir desde la planeación estratégica una propuesta de valor compartido permite responder a las necesidades de la comunidad afectada. Así mismo, se evidenció que las actividades sociales generaron una relación de mutuo beneficio entre las partes.
\end{abstract}

Palabras clave: valor compartido, planeación estratégica, organización

\begin{abstract}
The intention of this article was to identify how the mining company DG1 links, from strategic planning and shared value, its business proposal to the interested community. The methodology used in the research was qualitative, descriptive, not experimental. The results found established that defining a shared value proposal from strategic planning allows responding to the needs of the affected community. Likewise, it was evident that social activities generated a mutually beneficial relationship between the parties.
\end{abstract}

Key words: shared value, strategic planning, organization

\section{Introducción}

Las empresas se encuentran en un mundo donde la rapidez de los negocios, la volatilidad de los mercados, el cliente que constantemente es cambiante en sus decisiones y los altos niveles de incertidumbre son factores que

\footnotetext{
${ }^{1}$ Coordinador MBA administración. Universidad Católica Luis Amigó. mauricio.bedoyaji@amigo.edu.co

2 Docente investigadora. Facultad de ciencias Administrativas, Económicas y Contables. Universidad Católica Luis Amigó.

Jormaris.martinezgo@amigo.edu.co

${ }^{3}$ Docente investigador. Facultad de Comunicación, Publicidad y Diseño. Universidad Católica Luis Amigó. Juan.lopezme@amigo.edu.co

${ }^{4}$ Docente investigador. Facultad de ciencias Administrativas, Económicas y Contables. Universidad Católica Luis Amigó. Luis.quinterora@amigo.edu.co
} 
determinan que gerentes y directivos de una organización estén atentos para definir estrategias que les permitan responder al entorno empresarial donde compiten.

Desde la alta dirección Hamann (2003) analiza que se deben diseñar estrategias que respondan al entorno actual y prever situaciones del futuro cercano, análisis que se deben realizar con base en estudios propios del entorno, el mercado, la competencia y al interior de la misma empresa, con el fin de estar preparados para esos momentos complejos y cambiantes, contando con un plan diseñado a responder ante cualquier situación.

En el contexto empresarial, cada unidad productiva confronta realidades diferentes, donde el gerente debe dar respuesta a la toma de decisiones argumentada en datos, cifras e información objetiva que permita alcanzar las metas y objetivos propuestos por la alta dirección. Para el caso de las empresas mineras, el estudio del contexto es fundamental para definir el rumbo de la organización con certeza, dejando espacio mínimo para la incertidumbre. Este sector productivo tiene que cumplir con diferentes normatividad de índole nacional, regional y local, así como dar respuesta a las necesidades de la comunidad para llevar a cabo el desarrollo de un proyecto minero (Muñoz-Martín,2013).

Autores como Díaz (2015), Vives (2012) y Viveros (2016) señalan que las empresas deben de estar atentas a los cambios en el entorno, de manera especial en temas normativos y legales. Un directivo bien informado tiene menos probabilidad de equivocarse en la toma de decisiones, es por eso que al momento de decidir algún cambio en la organización se debe contar con un criterio objetivo y con datos reales.

Lo anterior permite evidenciar que es necesario que las empresas, en especial del sector minero, tengan en cuenta que la toma de decisiones debe estar acompañada de estrategias y objetivos, donde el valor compartido y la planeación estratégica aportan para orientar proyectos de corto y mediano plazo, así como en la orientación de la organización y responder a las necesidades de los grupos de interés, en especial de la comunidad donde el proyecto se desarrolla.

Con la realización de la investigación se pretende responder a las siguientes preguntas surgidas desde la gerencia de la empresa DG1: ¿Cómo se articula el valor compartido en los planes estratégicos de las empresas mineras productoras de metales localizadas en la ciudad de Medellín? así mismo se busca conocer: ¿Qué aspectos considera la empresa DG1 para vincular el valor compartido en sus actuaciones empresariales? ¿Qué importancia tiene definir estrategias desde el valor compartido y la planeación estratégica para que la empresa DG1 genere una transformación empresarial en el desarrollo de los proyectos mineros que vaya a ejecutar?

En el desarrollo de la investigación cuyo objetivo fue identificar cómo la empresa minera DG1 podía impactar de forma positiva a la comunidad en el desarrollo del proyecto minero, realizado en el sector de hatillo entre los Municipios de Medellín, Copacabana y Girardota, se analizaron dos categorías principales: el proyecto productivo y el impacto a la comunidad. Para eso se crearon mesas de trabajo y análisis de lo social y espacial de la ubicación de toda la comunidad que está en la zona del proyecto, con el fin de diseñar estrategias para llevar a cabo el trabajo y facilitar todo el proceso de adaptación de la comunidad a los nuevos cambios que se presentarán en la zona de impacto.

\subsection{Valor compartido}

De acuerdo con Porter y Kramer (2011), el valor compartido está determinado, por unas acciones que una empresa puede realizar con el fin de dar respuesta a situaciones sociales, orientando sus esfuerzos a brindar soluciones de acuerdo a las realidades de cada negocio, buscando así tener una articulación entre las actividades productivas y la responsabilidad social, más allá de lo que determinan las acciones éticas que una empresa debe cumplir en su objeto social. 
Buckland y Murillo (2014) precisan que el valor compartido tiene un vínculo directo con la innovación social, es decir, aparecen dos conceptos que se asocian y se apoyan para clarificar aspectos de la forma en que las empresas responden a los compromisos sociales y económicos de la comunidad. Su énfasis radica, según Díaz (2015), en la forma en que cada uno de los actores, empresa y comunidad, se apoyan y brindan un vínculo fuerte para que entre estas sea coherente la forma en que responde a sus diferentes responsabilidades. Si bien desde la organización debe pensarse en el valor que comparte con sus grupos de interés, estos también deben aportar a la organización para gestar una codependencia, o mejor, un comensalismo en cualquiera de los tres frentes: social, ambiental o financiero.

Según el artículo realizado por Méndez y Gómez (2017), las compañías son un agente económico importante en el desarrollo de un país, ya que estas generan empleo y permiten mejorar la calidad de vida de sus ciudadanos, es así, como el valor compartido tiene una relación directa en la actuación social de la empresa y la productividad que esta tiene definida para responder a sus necesidades internas y externas. Pero surge también la pregunta ¿Cada acción social es valor compartido?

Vélez (2015) explica el concepto de valor compartido, vinculado con la sostenibilidad, buscando un equilibrio entre las actuaciones empresariales, el entorno social y el medio ambiente, donde el equilibrio entre lo económico y los recursos naturales, tiene un impacto importante en la reputación de una compañía, que permita un desarrollo favorable, tanto en lo financiero como en resultados sobre la conservación de territorios y comunidades.

Como podemos analizar, las definiciones sobre el valor compartido son diversas y se interpretan de acuerdo a la forma en que una empresa puede aplicar este tipo de estrategias empresariales. Se deja claro, que el valor compartido debe tener trascendencia dentro de la organización y que todos los colaboradores tengan claro que en la organización producir y generar utilidades es el medio pero no el fin, por eso la alta dirección debe contemplar este tipo de estrategias válidas para implementar en las empresas que estén orientadas a entregarle al mercado productos y servicios de manera integral, respondiendo a necesidades propias del mercado.

En la figura 1 se ilustra la relación entre la empresa y sus grupos de interés. El valor compartido se evidencia en tanto esté dentro de un contexto real y pertinente para la organización y sus grupos de interés, allí entonces, la empresa propone la creación de valor, mientras que los interesados, aportan ideas que se convierten en acciones estratégicas que los compromete a la creación conjunta de valor.

Figura 1

Enlace valor compartido empresa y comunidad

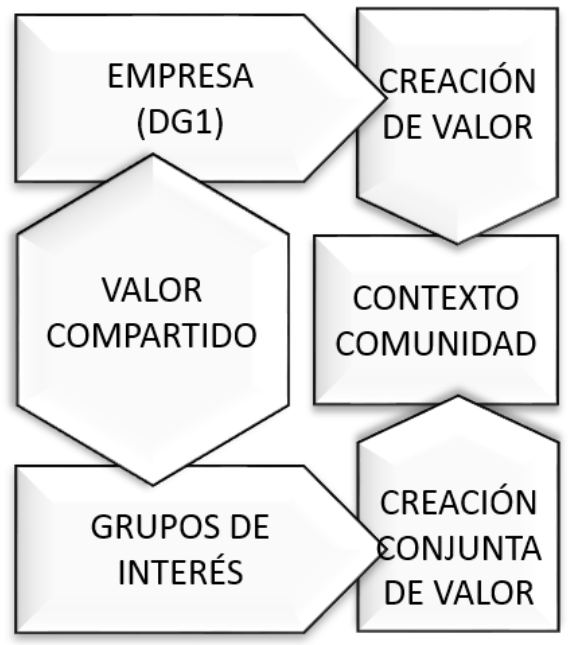

Fuente: Elaboración propia (2019) 
La anterior figura, demuestra lo valioso que resulta que la empresa DG1, vincule la creación de valor y valor compartido, esto permite articular las fortalezas que tiene la empresa, luego se agrupa el contexto con la comunidad y los grupos de interés, esto detalla de manera directa la articulación que se debe establecer con los grupos de interés; para luego definir una creación conjunta de valor que asocia de manera integral todos los procesos estratégicos que tiene la empresa para dar respuesta a sus actuaciones en el medio empresarial.

\subsection{Planeación estratégica}

Las empresas actuales de los diversos sectores económicos, tales como el sector agrícola, la industria y los servicios, evalúan cómo deben dar respuesta a procesos que les permita atender diferentes necesidades, tanto internas como externas, es así como, una empresa debe orientar sus esfuerzo a consolidarse en el mercado, buscando fortalecerse en el servicio, pero todo debe estar enmarcado desde la creación de la empresa, es decir, centrar en todo el proceso de la planeación estratégica definiendo los intereses propios, pero siendo responsable con lo que ofrecen al mercado y respondiendo a la sostenibilidad del medio ambiente (Franco y Henao, 2015).

Drucker (2002) considera que el líder de una compañía debe estar preparado para llevar a cabo una gestión administrativa de manera eficiente, eficaz y que lleve a la organización a cumplir los logros que se propone, teniendo criterio y conocimiento claro que la empresa es un agente dinámico que busca consolidar su razón de ser de adentro hacia afuera, determinando así su actuar en el presente y futuro.

Asímismo, Zapater (2017) estima que la empresa debe trazar sus planes bajo unas realidades concretas, debe realizar su planeación estratégica bajo realidades políticas, legales, sociales, ambientales y tecnológicas; igualmente desarrollar planes a corto, mediano y largo plazo, que logren orientar cada uno de los esfuerzos que la empresa tiene para cumplir sus metas y objetivos propuestos. Lograr lo anterior, requiere de asignación de tres elementos, lo financiero, logístico y humano, para luego terminar con indicadores que midan la gestión realizada en cada uno de los planes.

Igualmente, Porter (1996) explica que el proceso de planeación estratégica logra orientar a la organización a considerar aspectos de inversión, transformación, adaptación y cambio para ir en una búsqueda de una ventaja competitiva, es decir, ser el mejor de la industria y lograr niveles de eficiencia y eficacia, que le permita trabajar de manera coordinada en procesos de cambio constantes, analizando la competencia, el mercado y los clientes, estos últimos como el eje central de la planeación estratégica.

Por su parte, Araya (2017) define la planeación estratégica como un medio para lograr los objetivos que una empresa se traza, que debe ajustarse de acuerdo a comportamientos, tanto interno como externo, desde lo interno considerar lo que puede ser mejorado dentro de la empresa con el fin de responder a las necesidades del medio externo, y desde lo exógeno estar atento a los cambios legales, políticos, sociales, culturales, ambientales y tecnológicos.

En conclusión, de acuerdo a cada uno de los autores referenciados anteriormente, se expresa que los diferentes conceptos acerca de la planeación estratégica, logra integrar elementos puntuales, como son metas, objetivos, estrategias, tácticas y recursos, pero que todas terminan orientando y afinando lo que significa determinar el rumbo de una empresa para lograr lo que se propone dentro de su objeto social.

\section{Metodología}

La definición teórica el proyecto de investigación se realizó desde las lógicas del paradigma interpretativo, con un enfoque cualitativo, es decir comprender las realidades de la comunidad en el desarrollo del proyecto e interpretar lo que la comunidad espera recibir desde la operación técnica y la zona de influencia. 
La investigación tuvo un diseño no experimental ya que no se manipulan variables, de tipo transversal, es decir se realizó la investigación en un solo momento; así mismo, se definió un alcance de tipo descriptivo, es decir la realidad del fenómeno y su asociación con la literatura (Hernández, Fernández y Baptista, 2014).

En la metodología utilizada se desarrollaron dos métodos de tipo cualitativo, el interaccionismo simbólico y la participación acción. Para la recolección de la información se utilizaron entrevistas, grupos focales y técnicas interactivas.

En el procesamiento de la información, a partir de los instrumentos aplicados, se trabajaron dos categorías, estas son la categoría espacial y la categoría social, la selección de estas categorías, se determinó por el propio impacto del proyecto minero a desarrollarse en la zona de influencia.

Entre los criterios de inclusión para el desarrollo de la investigación, se tomó en cuenta la constitución legal de la empresa, su razón social, el tiempo que lleva en el mercado y el desarrollo de un proyecto minero en una zona semi rural, donde la comunidad no conocía el por qué una empresa minera podía extraer el oro y ellos no podían hacerlo de forma artesanal. La empresa desde su objeto social tiene dentro de sus funciones diferentes actividades relacionadas con la exploración, explotación y aprovechamiento de recursos naturales, así mismo, tiene unidades de negocio que están enmarcadas en los procesos de construcción de vías secundarias y tercerías, construcción de muros de contención y desarrollo de pavimentación de vías.

Para el desarrollo de la información se establecieron las siguientes categorías, en la tabla 1, se detallan cada una de ellas.

Tabla 1

Categorías de análisis

\begin{tabular}{|c|c|c|}
\hline \multirow{4}{*}{ Categoría } & \multicolumn{2}{|c|}{ Subcategoría } \\
\hline \multirow{4}{*}{ Concepto } & 1 & Comunidad \\
\cline { 2 - 3 } & 2 & Grupos de interés \\
\cline { 2 - 3 } & 3 & Medio Ambiente \\
\cline { 2 - 3 } & 4 & Valor compartido \\
\cline { 2 - 3 } & 5 & Planeación estratégica \\
\hline \multirow{4}{*}{ Factores de } & 1 & Identificación de actores \\
\cline { 2 - 3 } & 2 & Relacionamiento comunitario \\
\cline { 2 - 3 } & 3 & Aspectos sociales \\
\cline { 2 - 3 } & 4 & Aspectos espaciales \\
\hline
\end{tabular}

Fuente: Elaboración propia a

partir de encuestas (2019)

\section{Resultados}

En los resultados encontrados en el desarrollo del proyecto de investigación realizado con la empresa minera DG1, se identificó la necesidad de aplicar la matriz de debilidades, oportunidades, fortalezas y amenazas - DOFA, no solo desde lo corporativo, sino desde lo operativo, es decir, realizar una propuesta integral para poder dar respuesta al proyecto teniendo en cuenta el direccionamiento estratégico, el desarrollo operativo del proyecto y el impacto en la comunidad. Así mismo, se identificó que la extracción minera se desarrolla en tres unidades de negocio que pretenden impactar y participar en mercados diferentes. Esta configuración orgánica llevó a decidir si las propuestas de generación de valor conjunto se darían para cada unidad de negocio, o si se plantearían de manera integrada. 
La consultoría estableció entonces, luego de revisar y confrontar la matriz DOFA de cada unidad de negocio que, para dar más claridad a las particularidades de operación, cada unidad de negocio realizaría su análisis y valoración cruzada de fortalezas, debilidades, oportunidades y amenazas. La consultoría realizó una comparación transversal de las tres matrices para identificar cuándo una misma acción pudo considerarse fortaleza para una unidad de negocio y al mismo tiempo, debilidad para otra, igualmente sucedió con las oportunidades y amenazas. Esto permitió identificar con claridad dentro de la matriz ponderada, qué acciones estratégicas darían. Las acciones descritas en las matrices no pueden ser presentadas en los resultados debido a cláusulas de confidencialidad en la consultoría.

Además del ejercicio anterior, se propuso a la empresa DG1, el desarrollo de talleres y mesas de trabajo con la comunidad, para analizar aspectos que se pudieran establecer como hoja de ruta para acercarse a todas las necesidades que tienen y cómo dar respuesta desde la relación mutua y gana-gana para la creación conjunta de valor, qué potencial puede aportar la comunidad a la cadena de valor de DG1.

De dichos talleres y mesas, se hizo evidente el interés de la comunidad para desarrollar en conjunto una estrategia de valor compartido ajustado a las necesidades propias de la zona de influencia y que la organización pueda realizar la explotación minera en la zona geográfica determinada con la participación productiva de la comunidad en algunos procesos o etapas de la cadena de valor.

La categoría espacial determinó que para el área de influencia tanto directa e indirecta que tienen relación con el proyecto, ofrecerá unas condiciones favorables para la empresa, así como el desarrollo mismo de la exploración minera, la sensibilización en la zona, logró aclarar aspectos que se presentaban confusos, así la explotación minera se realizará con el apoyo de la comunidad.

Las propuestas de acciones estratégicas halladas se identificaron a partir de la construcción de una tabla básica que sirvió para establecer nuevos relacionamientos con los grupos de interés y de acuerdo con esto, proponer estrategias para la generación de valor conjunto. Para efectos de esta publicación, se presenta la tabla vacía con el fin de respetar la confidencialidad de las estrategias para DG1.

Tabla 1

Relación estratégica de grupos de interés

\begin{tabular}{|l|l|l|l|}
\hline \multicolumn{1}{|c|}{ Grupos de interés } & Debilidades & Fortalezas & Propuesta estratégica \\
\hline Junta directiva & & & \\
\hline Empleados & & & \\
\hline Accionistas & & & \\
\hline Sindicato & Oportunidades & Amenazas & Propuesta estratégica \\
\hline Proveedores & & & \\
\hline & & & \\
\hline $\begin{array}{l}\text { Cliente intermedio } \\
\text { (distribuidores, } \\
\text { certificaciones, etc) }\end{array}$ & & & \\
\hline Cooperativa (s) & & & \\
\hline Ong y fundaciones & & & \\
\hline Zona de influencia & & & \\
\hline Admón pública & & & \\
\hline Competencia & & & \\
\hline Clliente final & & & \\
\hline
\end{tabular}

Fuente: Elaboración propia (2019)

Al organizar los diversos grupos de interés en categorías DOFA, tanto la organización como los grupos dejan claro cuál es el reto estratégico por asumir para la generación conjunta de valor compartido 
Las propuestas resultantes deberán ser contrastadas con la disponibilidad presupuestal y financiera de DG1, para definir aquellas acciones que se pueden ejecutar a corto plazo y para identificar posibles aliados que favorezcan la consecución de nuevos recursos para la generación de valor.

El direccionamiento estratégico a la generación de valor conjunto, estará entonces concentrando esfuerzos para mejorar las relaciones con los clientes intermedios (básicamente empresas de transporte y servicios logísticos), el establecimiento de convenios con cooperativas que faciliten la articulación entre cliente interno y zona de influencia y la diversificación de algunas ofertas de DG1 para un mercadeo extensivo que procure nuevos clientes finales en nuevas líneas de negocio desatendidas por las empresas competidoras.

En los resultados encontrados desde lo económico y social, se determinó que proyectos sostenibles y sustentables, si se pueden realizar, esto conlleva que las partes trabajen de manera coordinada, con el fin de dar respuesta a dificultades que se puedan presentar, el dialogo y la negociación arrojan buenos resultados para que ambas partes salgan beneficiadas y se logre tener una confianza mutua para este tipo de proyectos mineros.

\section{Conclusiones}

Las empresas deben estar al tanto de cómo realizar la mejor gestión para poder mantener la empresa en los contextos actuales. Hoy, las empresas del sector minero, en este caso del sector metales preciosos, deben de contar con las comunidades, ya que estas exigen que este tipo de empresas respondan a las mejores prácticas ambientales y sociales para el desarrollo de proyectos mineros.

Toda empresa debe ir en la búsqueda del éxito partiendo desde lo financiero, social y ambiental; la contribución de la empresa DG1 a la economía regional y local, se determinó por la generación de impacto que favoreció a la comunidad. Lo anterior, aporta a la calidad de vida de estas personas y para que la empresa pueda desarrollar sus actividades mineras con tranquilidad, pensando en alcanzar una relación ganar-ganar con todos los actores sociales, políticos, ambientales que intervienen en este tipo de proyectos mineros.

Asimismo, el desarrollo del proyecto es el primero que la empresa realiza con una zona de impacto en una comunidad, esto le permitió a DG1 identificar cómo prepararse y qué temas enfrentar para posteriores proyectos mineros en otras regiones de Antioquía y Colombia.

Igualmente, esta investigación tuvo un componente importante y es que la comunidad percibió que la empresa tenía el compromiso no solo de desarrollar el proyecto, sino de dejar para el futuro proyectos económicos productivos, mejorar las condiciones de habitabilidad, acceso y educativas, que permitieran que posteriores generaciones pudieran aprovechar lo realizado por la empresa.

En Antioquía y parte de Colombia, la minería ilegal está generando problemas sociales complejos como el desplazamiento, asesinatos, violencia e ingresos ilegales, que hace que la economía de una zona se vea afectada por un fenómeno que el Estado debe atacar. En este proyecto que tenía todos los permisos necesarios, se dio un resultado positivo y beneficioso para que la comunidad y la empresa generaran a su manera las mejores ganancias del trabajo en equipo.

Incorporar el valor compartido como filosofía y práctica de la planeación estratégica, puede resultar en la mejor manera de optimizar recursos y procesos, ceder parte de la responsabilidad de la cadena de valor a un aliado de los grupos de interés, puede hacer más flexible la operación productiva y fortalecer la reputación de marca. Para el caso de DG1, que busca cimentarse en un mercado primario, las propuestas de valor compartido son una oportunidad para innovar en procesos y productos, que diversifiquen la oferta entre sus clientes finales y le brinde mejores probabilidades de expansión con alternativas de producto aún no contempladas por su competencia. 
Los encadenamientos a través de proyectos productivos coexistentes con la minería son desarrollados por las empresas tomando en cuenta regulaciones de protección del medio ambiente y la comunidad, cada vez vienen tomando más protagonismo. A través de estos proyectos se están fortaleciendo las capacidades empresariales en las comunidades por medio de temas formativos para estructurar proyectos sostenibles, estos pueden se agrícolas, piscícolas, apícolas y de abastecimiento de suministros para las operaciones mineras. Adicionalmente, algunos de estos tienen como foco las mujeres líderes y emprendedoras, "se trabaja con las mujeres de la región para crear fuentes de ingresos diferentes a la minería" expresó uno de los entrevistados.

\section{Referencias bibliográficas}

Araya, A. (2017). Modelos de planeación estratégica en empresas familiares. Revista Tec Empresarial, 11, (1), 23-34. Recuperado de: https://www.scielo.sa.cr/pdf/tec/v11n1/1659-3359-tec-11-01-00023.pdf

Buckland, H y Murillo, D. (2014). La innovación social en América Latina. Recuperado de: http://proxymy.esade.edu/gd/facultybio/publicos/1431613105032_ESADE-FOMIN-La-innovacion-socialen-America-Latina-Marco-conceptual-y-agentes-1.pdf

Díaz, N. (2015). La creación de valor compartido: estrategia de sostenibilidad y desarrollo empresarial. Revista cultura Latinoamericana, 22, (2), 207-230. Recuperado de: https://editorial.ucatolica.edu.co/ojsucatolica/revistas_ucatolica/index.php/RevClat/article/view/1629/15 05

Drucker, P. (2002). La gerencia en la sociedad futura. Bogotá: Norma.

Franco, G., y Henao C. (2015). Implementation of strategic planning to mining in Colombia. Aplicación de la planificación estratégica a la minería en Colombia. Boletín de Ciencias de La Tiierra, (37), 20-24. Recuperado de: https://doi.org/10.15446/rbct.n37.48557

Hamann, R. (2003). Mining companies' role in sustainable development: The "why" and "how" of corporate social responsibility from a business perspective. Development Southern Africa, 20(2), 237-254. Recuperado de: https://doi.org/10.1080/03768350302957

Hernández, R. Fernández, C. y Baptista, M. (2014). Metodología de la investigación. Bogotá:Mc Graw Hill.

Méndez, M y Gómez, M. (2017). Factores incidentes para crear valor compartido en las Mipymes de Bogotá. Revista Suma de Negocios, 8, (18), 96 - 105. Recuperado de: https://www.sciencedirect.com/science/article/pii/S2215910X17300253

Muñoz-Martín, J. (2013). Ética empresarial, Responsabilidad Social Corporativa (RSC) y Creación de Valor Compartido (CVC). Journal Globalization, Competitiveness and Governability, 7(3). Recuperado de: https://doi.org/10.3232/GCG.2013.V7.N3.05

Porter, M. (1996). Ventaja competitiva. Madrid, España: Pirámide.

Porter, M. y Kramer, M. (2011). La creación de valor compartido. Harvard Business Review, pp. 4 - 18.

Vélez Rolón, A. (2015). Formación de estudiantes de administración Para generar valor compartido en el sector empresarial desde el marco de la sostenibilidad. Revista Espacios, 36,(21). Recuperado de: https://www.revistaespacios.com/a15v36n21/153621E2.html\#uno

Vives, A. (2012). Compartir el Valor Creado versus Crear Valor Compartido. Revista Cuadernos Latinoamericana de administración. VII, (14), 5-7. Recuperado de: http://www.redalyc.org/pdf/4096/409634368001.pdf 
Viveros, H. (2016). Examining Stakeholders' Perceptions of Mining Impacts and Corporate Social Responsibility. Corporate Social Responsibility and Environmental Management, 23(1), 50-64. Recuperado de: https://doi.org/10.1002/csr.1363

Zapater, L. (2017). Análisis de las falacias en torno a la teoría de la Soberanía Nacional (O Popular). Revista Prolegómenos, 20, (39), 39-54. Recuperado de: https://doi.org/10.18359/prole.2722

Esta obra está bajo una Licencia Creative Commons Attribución-NoCommercial 4.0 International

\section{(cc) BY-NC}

